\title{
Гармонический анализ колебательных спектров твердых тел и его применение при расчете функций состояния минералов
}

\author{
Антонов А.А. \\ ФИЦ КНЦ РАН, Anamumbl, z8906290@yandex.ru
}

\begin{abstract}
Аннотация. Колебательной составляющей термодинамических потенциалов и энтропии отводится особая роль при статистических расчетах: именно она обуславливает температурную зависимость функций состояния в интервалах температур, находящихся между точками фазовых переходов. В статье приведен вывод формул, пригодных для представления квазинепрерывного колебательного спектра, заданного в табличной форме, тригонометрическим рядом с любой желаемой точностью. Результаты могут быть полезны, в частности, для определения теплоемкости редких минералов как функции от температуры без выполнения большого числа калориметрических измерений, - по их колебательным спектрам.

Ключевые слова: статистическая термодинамика, ИК-спектроскопия, комбинационное рассеяние, термический анализ, функции состояния, числовые ряды, дискретное преобразование Фурье.
\end{abstract}

\section{Harmonic analysis of solid substances vibration spectra and its application for calculations of mineral species state functions}

\author{
Antonov A.A. \\ FRC KSC RAS, Apatity, z8906290@yandex.ru
}

\begin{abstract}
A special role is assigned to the vibration part of thermodynamic potentials and entropy in the statistic calculations, since it determines the temperature dependence of state functions in temperature ranges between phase transition points. This paper provides the derivation of basic formulas for representation of quasicontinuous vibration spectrum specified in a tabular form by a trigonometric series with any desired accuracy. In particular, the results can be useful to determine rare minerals heat capacity as a function of temperature using their vibration spectra, without numerous calorimetric measurements.
\end{abstract}

Keywords: statistical thermodynamics, IR spectroscopy, Raman spectroscopy, thermal analysis, state functions, numerical series, discrete Fourier transform.

\section{Введение}

Для уверенного получения синтетических аналогов минеральных видов необходимо сделать правильный выбор как состава исходной композиции, так и значений параметров состояния, определяющих состояние системы, при котором формируется целевой продукт. Качественный состав исходной композиции можно с ограниченной надежностью определить на основе многочисленных качественных подходов. Для определения как количественного состава, так и значений параметров состояния, при которых целевой процесс становится возможным, требуется проведение термодинамических расчетов. Последнее возможно в том случае, если известны значения энтропии и изменения одного из термодинамических потенциалов образования целевого продукта, для определения этих значений наиболее простым является подход. основанный на предположении о равенстве энергий связей в рассматриваемом соединении и в более простом, для которого функции состояния известны. Такой подход оправдан, если все эти соединения имеют схожую структуру; в противном случае получаются неудовлетворительные результаты. Помимо этого, для определения функций состояния твердых веществ можно использовать результаты статистического расчета, вводя поправку на межмолекулярное взаимодействие, при отсутствии фазовых переходов не зависящую от T, по результатам определения теплоемкости в узком интервале температур. Этот подход делает возможным определение функций состояния редких минеральных видов с точностью, достаточной для получения синтетического аналога без термического разрушения исследуемого образца (минимальная масса, необходимая для термогравиметрии и дифференциальной сканирующей калориметрии составляет 5 мг.). При этом, если целевой продукт - неметалл при определении теплоемкости 
можно ограничиться только колебательной и вращательной составляющими, причем, последняя не зависит от $\mathrm{T}$ и равна $3 \mathrm{R}$ для изохорной теплоемкости, $4 \mathrm{R}$ - для изобарной без учета поправки на межмолекулярное взаимодействие, где $\mathrm{R}$ - универсальная газовая постоянная.

Целью работы послужила разработка универсального метода нахождения функции распределения колебаний по частоте с любой желаемой точностью.

Реализация цели потребовала решения следующих задач:

1. Вывод формул, позволяющих осуществить представление таблично заданной функции рядом Фурье;

2. Выбор подходящего критерия для оценки достоверности аппроксимации;

3. Разработка алгоритма нахождения числа членов в разложении при заданном значении критерия достоверности аппроксимации.

\section{Материалы и методы}

Колебательные спектры заимствованы из открытого источника [1], все расчеты выполнены при помощи коммерческого пакета компьютерной алгебры maple.

Как упоминалось выше, изобарную теплоемкость $\mathrm{C}_{\mathrm{p}}$ твердого тела, в молекулах которого отсутствуют внутренние вращения, можно выразить следующим образом [2]:

$$
C_{p}=C_{\text {вр. }}+C_{\text {кол. }}+\lambda
$$

или

$$
C_{p}=4 R+C_{\text {кол. }}+\lambda
$$

Поправку на межмолекулярное взаимодействие $\lambda$ можно найти по результатам дифференциальной сканирующей калориметрии в узком интервале температур:

$$
C_{p}=\frac{D S C(\text { Обр. }+ \text { Тигл. })-D S C \text { (Тигл. })}{D S C(\text { Ст. }+ \text { Тигл. })-D S C \text { (Тигл. })} \cdot \frac{n(\text { Ст. })}{n(\text { Обр. })} \cdot C_{p}(\text { Ст. })
$$

где DSC(Обр.+Тигл.), DSC(Ст.+Тигл.), DSC(Тигл.) - аналитический сигнал от образца с тиглем, от стандарта с тиглем и от пустого тигля соответственно;

$\mathrm{n}(\mathrm{Oбр.)}$ и $\mathrm{n}($ Ст. $)$ - количества вещества образца и стандарта соответственно;

$\mathrm{C}_{\mathrm{p}}$ и $\mathrm{C}_{\mathrm{p}}(\mathrm{C}$. $)$ - молярные теплоёмкости образца и стандарта соответственно.

Объединив (2) и (3), выразим $\lambda$ :

$$
\lambda=\frac{D S C \text { (Обр. }+ \text { Тигл. })-D S C \text { (Тигл. })}{D S C(\text { Ст. }+ \text { Тигл. })-D S C \text { (Тигл. })} \cdot \frac{n(\text { Ст. })}{n(\text { Обр. })} \cdot C_{p}(\text { Ст. })-4 R-C_{\text {кол. }}
$$

Далее мольную теплоёмкость исследуемого вещества как функцию от температуры можно найти из (2), не прибегая к термическому анализу:

$$
C_{p}(T)=C_{\text {кол. }}(T)+\text { const }=C_{\text {кол. }}(T)+4 R+\lambda
$$

Статистическая механика даёт следующее выражение для колебательной составляющей теплоёмкости (с/м, например, [3]):

$$
C_{\text {кол. }}=2 R T \frac{d \ln Q_{\text {кол. }}}{d T}+R T^{2} \frac{d^{2} \ln Q_{\text {кол. }}}{d T^{2}}
$$

Колебательная сумма по состояниям одной молекулы $\mathrm{Q}_{\text {кол. }}$ в гармоническом приближении равна:

$$
Q_{\text {кол. }}=\left(\frac{1}{1-\exp \left(-\frac{h \bar{v}}{k T}\right)}\right)^{s}
$$


где

$\mathrm{h}$ - постоянная Планка;

$\mathrm{k}$ - постоянная Больцмана;

$\bar{v}$ - частота одного колебания, усреднённая по всем возможным состояниям;

$\mathrm{s}$ - число колебательных степеней свободы, для нелинейной молекулы s=3n- $\left(6 / \mathrm{N}_{\mathrm{a}}\right) \approx 3 \mathrm{n}(\mathrm{n}$-число атомов в молекуле).

Средняя частота одного колебания представляет собой среднее статистическое:

$$
\bar{v}=\int_{0}^{v_{\max }} v \cdot g(v) d v
$$

Для нахождения функции распределения $g(v)$ колебаний по частоте представим весь спектр тригонометрической формой ряда Фурье. Проблема состоит в том, что известные формулы ЭйлераФурье предназначены для разложения функций, заданных аналитически. Для неотрицательных функций произвольного периода они имеют вид:

$$
\begin{gathered}
a_{0}=\frac{1}{l} \int_{0}^{2 l} f(x) d x \\
a_{n}=\frac{1}{l} \int_{0}^{2 l} f(x) \cos \frac{\pi n x}{l} d x \\
b_{n}=\frac{1}{l} \int_{0}^{2 l} f(x) \sin \frac{\pi n x}{l} d x \\
f(x)=\frac{a_{0}}{2}+\sum_{n=1}^{X}\left(a_{n} \cos \frac{\pi n x}{l}+b_{n} \sin \frac{\pi n x}{l}\right)
\end{gathered}
$$

Они могут быть обобщены на непериодическую функцию, если считать периодом всю область определения.

\section{Результаты и обсуждение}

Формулы (9) могут быть обобщены на таблично заданную функцию, коей по сути является ИК-спектр или спектр комбинационного рассеяния, если вспомнить, что определённый интеграл по своему определению является пределом интегральной суммы:

$$
\int_{0}^{2 l} f(x) d x=\lim _{n \rightarrow \infty} \sum_{i=1}^{n} f\left(c_{i}\right) \Delta x_{i}
$$

При анализе колебательных спектров удобно принять:

$$
\begin{array}{cc}
c_{1}=v_{2} & \Delta x_{1}=v_{3}-v_{1} \\
c_{2}=v_{4} & \Delta x_{2}=v_{5}-v_{3} \\
\ldots & \cdots \\
c_{i}=v_{2 i} & \Delta x_{i}=v_{2 i+1}-v_{2 i-1}
\end{array}
$$

Формулы (9) с некоторой точностью, зависящей от X, примут следующий вид: 


$$
\begin{gathered}
l=v_{N=2 i}-v_{2} \\
a_{0}=\frac{1}{2 l} \sum_{k=2}^{N=2 i} I_{k}\left(v_{k+1}-v_{k-1}\right) \\
a_{X}=\frac{1}{2 l} \sum_{k=2}^{N=2 i} I_{k}\left(v_{k+1}-v_{k-1}\right) \cos \frac{\pi X v_{k}}{l} \\
b_{X}=\frac{1}{2 l} \sum_{k=2}^{N=2 i} I_{k}\left(v_{k+1}-v_{k-1}\right) \sin \frac{\pi X v_{k}}{l} \\
I(v)=\frac{a_{0}}{2}+\sum_{i=1}^{X}\left(a_{X} \cos \frac{\pi X v}{l}+b_{X} \sin \frac{\pi X v}{l}\right)
\end{gathered}
$$

где $\quad v$ - частота колебания;

I - интенсивность колебания;

$\mathrm{X}$ - количество членов в разложении;

коэффициент $1 / 2$ в формулах для вычисления коэффициентов Фурье преобразует $\mathrm{k}=(2,4,6, \ldots 2 \mathrm{i})$ в $\mathrm{k}=(1,2,3,4 \ldots)$. Это становится возможным благодаря тому, что спектр снимается через равные интервалы волновых чисел.

Для оценки точности аппроксимации спектра тригонометрическим рядом нами выбран коэффициент детерминации:

$$
R^{2}=1-\frac{\sum_{k=1}^{N}\left(I_{k}-I\left(v_{k}\right)\right)^{2}}{\sum_{k=1}^{N}\left(I_{k}-\bar{I}\right)^{2}}
$$

Аппроксимации спектра тригонометрическим рядом с любой заданной точностью z в maple реализуется в виде цикла:

for $X$ from $s$ to $N$ do

$I:=$ “формулы 11 ”;

$R 2:=$ "формула 12 ”;

if $R 2>z$ then break;

end if;

end do: $X, R 2, I$

Полученное аналитическое выражение для интенсивности линий в спектре может быть легко преобразовано в функцию распределения колебаний по частоте, если применить условие нормировки:

$$
\int_{0}^{N} a \cdot I(v) d v=1 \stackrel{\rightrightarrows}{\Rightarrow} g(v)=a \cdot I(v)
$$

$\mathrm{a}-$ постоянный множитель, который находится из условия нормировки.

В заключение приведём спектр комбинационного рассеяния пирита [1], снятый при стандартных условиях, и картину, построенную по аналитическому выражению, полученному предлагаемым в настоящей работе способом с коэффициентом детерминации 0.9 (рис. 1). Так как сами аналитические выражения интенсивности линий в спектре как функции волнового числа и функции распределения колебаний по частоте имеют чрезвычайно громоздкий вид, в тексте данной работы они не приводятся. 


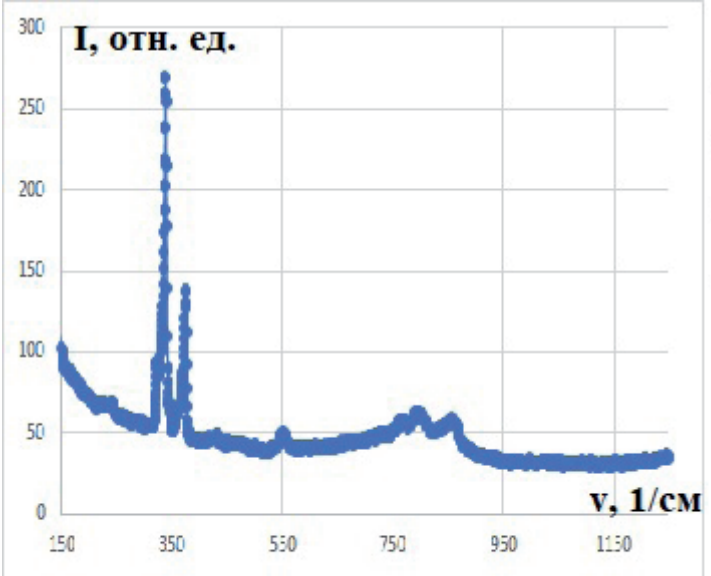

(a)

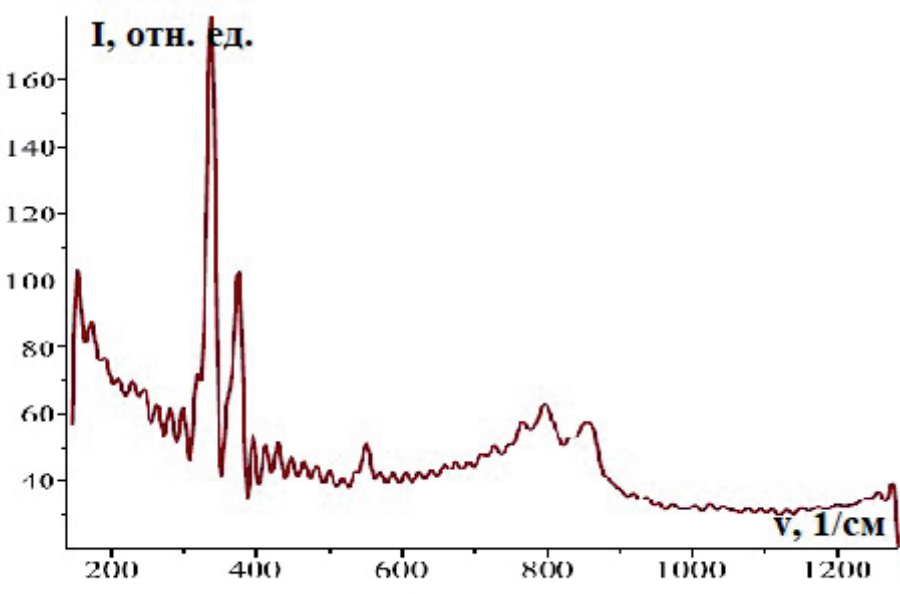

(б)

Рис. 1. Фактическая картина спектра комбинационного рассеяния $\mathrm{FeS}_{2}$ (а) и картина, построенная по аналитическому выражению I(v) в maple (б), полученному описанным в данной работе методом с $\mathrm{R}^{2}=0.9$.

Fig. 1. The factual picture of the spectrum in the Raman spectroscopy of $\mathrm{FeS}_{2}$ (a) and picture constructed using the analytical expression $\mathrm{I}(\mathrm{v})$ in maple (b) produced by the method described in this paper with $\mathrm{R}^{2}=0.9$.

Благодарности. Автор выражает признательность Андрею Олеговичу Калашникову (ГИ КНЦ РАН) за предоставленный для проведения расчётов высокопроизводительный компьютер.

Исследования проводились в рамках научной темы ФИЦ КНЦ РАН 0186-2019-0011.

\section{Литература}

1. RRUFF. Database of Raman spectroscopy [Электронный ресурс]. - Режим доступа: rruff.info. - Загл. с экрана.

2. Годнев И.Н. Вычисление термодинамических функций по молекулярным данным. М. Государственное изд-во технико-теоретической литературы. 1956. 423 с.

3. Ландау Л.Д., Лившиц Е.М. Теоретическая физика: Учеб. пособ. для вузов в 10 томах. Т. V. Статистическая физика. Ч. 1. М.: ФИЗМАТЛИТ. 2002. 616 с. 